\title{
BMJ Open Use of prescription opioids among patients with rheumatic diseases compared to patients with hypertension in the USA: a retrospective cohort study
}

\author{
Sarah K Chen, ${ }^{1}$ Candace H Feldman, ${ }^{1}$ Gregory Brill, ${ }^{2}$ Yvonne C Lee, ${ }^{3}$ \\ Rishi J Desai, ${ }^{2}$ Seoyoung C Kim ${ }^{1,2}$
}

To cite: Chen SK, Feldman $\mathrm{CH}$, Brill G, et al. Use of prescription opioids among patients with rheumatic diseases compared to patients with hypertension in the USA: a retrospective cohort study. BMJ Open 2019;9:e027495. doi:10.1136/ bmjopen-2018-027495

\section{- Prepublication history and} additional material for this paper are available online. To view these files, please visit the journal online (http://dx.doi. org/10.1136/bmjopen-2018027495).

Received 25 0ctober 2018 Revised 29 May 2019 Accepted 5 June 2019

\section{Check for updates}

(c) Author(s) (or their employer(s)) 2019. Re-use permitted under CC BY-NC. No commercial re-use. See rights and permissions. Published by BMJ.

${ }^{1}$ Division of Rheumatology, Allergy and Immunology, Department of Medicine, Brigham and Women's Hospital, Harvard Medical School, Boston, MA, USA

${ }^{2}$ Division of

Pharmacoepidemiology and Pharmacoeconomics, Department of Medicine, Brigham and Women's Hospital, Harvard Medical School, Boston, MA, USA

${ }^{3}$ Northwestern University Feinberg School of Medicine, Chicago, Illinois, USA

Correspondence to

Dr Sarah K Chen;

schen30@bwh.harvard.edu

\section{ABSTRACT}

Objective Long-term opioid prescribing has increased amid concerns over effectiveness and safety of its use. We examined long-term prescription opioid use among patients with rheumatoid arthritis (RA), systemic lupus erythematosus (SLE), psoriatic arthritis (PSA) and ankylosing spondylitis (AS), compared with patients with hypertension (HTN).

Methods We used Truven MarketScan, a US commercial claims database (2003-2014) and identified RA, SLE, PsA and AS cohorts, each matched by age and sex to patients with HTN. We compared long-term opioid prescription use during 1 year of follow-up and used multivariable Poisson regression model to estimate the relative risk (RR) of receiving opioid prescriptions based on underlying disease cohort.

Results We identified 181710 RA (mean age 55.3 \pm 13.1 , $77 \%$ female), 45834 SLE (47.1 $13.1,91 \%$ female), 30

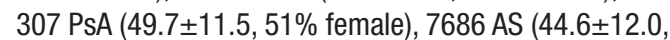
$39 \%$ female) and parallel numbers of age-matched and sex-matched patients with HTN. The proportion of patients receiving long-term opioid prescriptions, and other measures of opioid prescriptions were higher among rheumatic disease cohorts and highest in patients with AS. AS was associated with the highest RR of receiving longterm opioid prescriptions (RR $2.73,95 \% \mathrm{Cl} 2.60$ to 2.87 ) versus HTN, while RRs were 2.21 (2.16 to 2.25) for RA, 1.94 (1.87 to 2.00) for PsA and 1.82 (1.77 to 1.88) for SLE. Conclusions Patients with rheumatic disease have higher rates of long-term opioid prescriptions, and patients with AS have the highest risk of receiving opioid prescriptions versus patients with HTN. Further studies investigating the effectiveness of disease-targeted treatments on decreasing opioid use in these four rheumatic diseases may provide strategies for reducing prescription opioids.

\section{INTRODUCTION}

Prescription opioid use in the USA has increased, amid rising concerns over its effectiveness in treating non-cancer related pain, and safety of its use. ${ }^{2}$ Improvement in chronic non-cancer pain with opioid use may be minimal at best, ${ }^{3}$ and there is limited evidence on effectiveness of long-term opioid
Strengths and limitations of this study

- This was a large US nationwide study which examined rates of opioid prescribing in four rheumatic diseases (rheumatoid arthritis, systemic lupus erythematosus, psoriatic arthritis and ankylosing spondylitis) which are associated with pain as a common complaint and for which prescription opioid use patterns have not been extensively studied.

- We identified a comparison cohort of age-matched and sex-matched patients with hypertension to compare rates of opioid prescribing in patients with rheumatic diseases compared with patients without these conditions. Hypertension is a non-painful chronic condition with routine visits, but this patient population may not be representative of all patients without rheumatic diseases.

- Other limitations of this study include lack of information about disease severity, pain, race/ethnicity, cannabinoid use and physician characteristics which are covariates that may be associated with prescription opioid use.

use versus non-use for chronic non-cancer pain. ${ }^{4}$ However, several studies have demonstrated increased risk of harms with long-term opioid use. Adverse effects of dry mouth, nausea, constipation are common with opioid use, as well as increased risk of opioid abuse or dependence. ${ }^{56}$ Furthermore, the opioid epidemic has coincided with an alarming rise in opioid-related overdoses. ${ }^{78}$ Opioid use is additionally associated with increased risk of death, infections and fractures. ${ }^{7-12}$

Pain is one of the most common complaints for patients with rheumatic diseases, ${ }^{13-15}$ and therefore, patients with rheumatic diseases may have higher frequency of opioid use compared with the general population. Several studies have examined opioid use in patients with rheumatoid arthritis (RA), and found that the prevalence of opioid prescription dispensing is common in up to $40 \%$ 
among patients with RA compared with $24 \%$ in non-RA patients. ${ }^{16}{ }^{17}$ Opioid use in patients with RA has increased since the turn of the century, coinciding with the increase in opioid use in the general population. ${ }^{1} 1819$ However, rates of long-term prescription opioid use among patients with other rheumatic diseases such as systemic lupus erythematosus (SLE), psoriatic arthritis (PsA) and ankylosing spondylitis (AS) and comparison to those without rheumatic diseases have not been examined and may identify additional rheumatic disease populations at high risk of opioid use and related harms.

The aim of this study was to compare the use of longterm prescription opioids among patients with four common rheumatic diseases: RA, SLE, PsA and AS compared with age and sex-matched patients who have hypertension (HTN) but none of the four rheumatic diseases. We hypothesised that rates of long-term $(\geq 90$ days) opioid prescriptions would be higher among rheumatic disease cohorts compared with the HTN cohort. We also hypothesised that the relative risk of receiving long-term opioid prescriptions would be the highest for RA, which has been the focus of most studies regarding pain and opioid use and has an older patient population, as older age has been reported to be associated with prescription opioid use. ${ }^{2021}$

\section{METHODS}

\section{Data source and cohort definitions}

We used de-identified medical and pharmacy claims data from the Truven MarketScan database (2003-2014). This database contains longitudinal, comprehensive healthcare data from several different commercial managed care plans, representing mostly working individuals and their family members in the USA from all 50 states. ${ }^{22}$

We identified each rheumatic disease cohort (RA, SLE, PsA or AS) by using $\geq 2$ disease-specific International Classification of Diseases (ICD-9) codes, which were 7-365 days apart, and at least one dispensing for disease-specific medications on or after the second diagnosis code after a 12-month eligibility period (figure 1). ICD-9 codes and

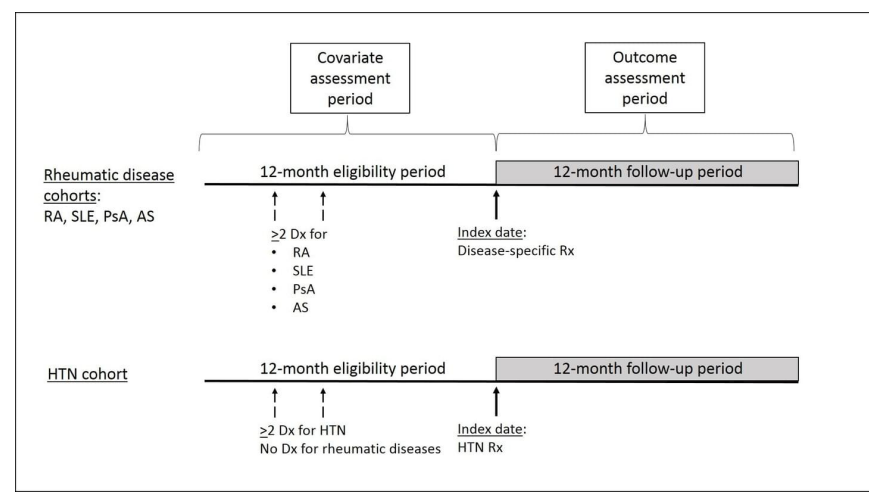

Figure 1 Cohort and study design diagram AS, ankylosing spondylitis; Dx, diagnosis; HTN, hypertension; PsA, psoriatic arthritis; RA, rheumatoid arthritis; Rx, prescription; SLE, systemic lupus erythematosus. disease-specific medications for each of the cohorts are listed in online supplementary table S1. The index date was defined as the date of disease-specific medication prescription fill when the cohort definition was met. The four rheumatic disease cohorts were mutually exclusive, and patients were enrolled into the cohort of the disease for which they first met the cohort definition.

We matched each rheumatic disease cohort patient by age at index date and sex to a patient with HTN, as it is a common non-painful, non-inflammatory, chronic condition. Patients with HTN have routine outpatient visits during which medications including opioids could be potentially prescribed and baseline covariates can be measured to minimise the risk of ascertainment bias, and has been used as a control group similarly in previous claims-based research. ${ }^{23}$ We defined HTN cohort status by at least 2 ICD-9 codes which were 7-365 days apart for HTN, and at least one anti-HTN medication prescription on or after the second diagnosis code after a 12-month eligibility period. The index date was defined as the date of anti-HTN medication prescription when the HTN cohort definition was met. HTN cohort patients required never having had a diagnosis of RA, SLE, PsA or AS during the study period.

We required all patients with rheumatic disease and HTN to be $\geq 18$ years of age at index date and to have a follow-up period for at least 12 months from and including the index date. We excluded patients with history of malignancy.

\section{Patient and public involvement statement}

This is a retrospective database study; patients were not directly involved in the research.

\section{Baseline covariate assessment}

During the 12-month eligibility period prior to the index date, we assessed baseline covariates including demographics (ie, age, sex, calendar year of index date and region of residence) and healthcare utilisation patterns (ie, number of outpatient visits, primary care visits, emergency department visits, hospitalisations and physical therapy use). We also assessed comorbid conditions including back pain, fibromyalgia, substance use, depression, migraine headache, osteoarthritis, fracture and surgery which are known risk factors for pain or opioid use, and other comorbidities including heart disease, kidney disease, chronic obstructive pulmonary disease, liver disease by ICD-9 codes and calculated combined comorbidity index ${ }^{24}$ at baseline. We assessed medication use of non-steroidal anti-inflammatory drugs (NSAIDs), COX-2 enzyme inhibitors (COXIBs), benzodiazepines, antidepressant use and gamma-aminobutyric acid (GABA) analogues. We measured baseline use of disease related medications including conventional synthetic and biologic disease-modifying antirheumatic drugs (DMARDs), and assessed baseline glucocorticoid use and cumulative glucocorticoid dose in $\mathrm{mg}$. We measured baseline use of opioids, naloxone and methadone. 


\section{Outcomes}

We measured long-term opioid prescriptions dispensed during the 1-year follow-up period from the index date as our primary outcome. Opioid medications are listed in online supplementary table S2. We defined long-term opioid prescriptions as cumulative opioid prescriptions dispensed adding up to $\geq 90$ days of opioid prescription during the 1-year follow-up period.

As our secondary outcome measures, we assessed any opioid prescriptions dispensed, receipt of $\geq 3$ types of opioid prescription, immediate-acting and extended-acting opioid in all rheumatic disease and matched HTN cohorts. We also calculated median cumulative morphine equivalent dose per day of opioid prescription (calculated as cumulative amount of opioids in milligrams (mg) in morphine equivalent dose divided by the number of days of prescription dispensing) during 1-year follow-up for patients receiving any opioid use in rheumatic disease and matched HTN cohorts using opioid morphine equivalent conversion factors per Centers for Disease Control and Prevention. ${ }^{25}$ We additionally assessed long-term opioid prescriptions after stratifying patients by baseline medication use of biologic DMARDs for RA, PsA and AS cohorts, and by the use of immunosuppressant medications other than hydroxychloroquine for SLE.

\section{Statistical methods}

We compared baseline characteristics of the four rheumatic disease cohorts and their matched HTN cohorts. We compared the proportion of patients receiving long-term, any use, >3 types, immediate-acting, extended-acting opioid and morphine equivalent dose per day opioid prescriptions in each rheumatic disease cohort to their matched HTN cohorts. We calculated the proportion of patients in each cohort receiving long-term opioid prescriptions for each calendar year of their index date and used linear regression to assess for significant trends in opioid use over the calendar years within each cohort.

We estimated relative risk of receiving long-term opioid prescriptions by using a Poisson regression model (PROC GENMOD), adjusting for cohort type (ie, RA, SLE, PsA, AS or HTN), age, sex, baseline comorbidities, medication use and healthcare utilisation patterns. The variables in our model were chosen a priori as potential factors associated with rheumatic diseases and receiving prescription opioids. To test for possible collinearity in our model, we ran a multicollinearity analysis to test for high correlation between the variables. Using a threshold of variation inflation factor $<10$ and tolerance $>0.1$, we found no evidence of multicollinearity in our model. ${ }^{26}$ In sensitivity analysis, we stratified patients with AS by baseline use of biologic DMARDs and estimated relative risk of receiving longterm opioid prescriptions compared with their matched HTN counterparts, adjusting for the same variables as in our main model.

All analyses were conducted using SAS V.9.4 (SAS Institute).

\section{RESULTS}

We identified 181710 patients with RA (mean age $55.3 \pm 13.1$ years, $77 \%$ female), 45834 patients with SLE (mean age $47.1+13.1$ years, $91 \%$ female), 30307 patients with PsA (mean age $49.7 \pm 11.5$ years, $51 \%$ female) and 7686 patients with AS (mean age $44.6 \pm 12.0$ years, $39 \%$ female) (table 1). Each matched HTN cohorts was composed of an equal number of patients with the same mean age and sex distribution as their respective rheumatic disease cohort.

The presence of baseline smoking, alcohol and substance use was similar across all cohorts. Fibromyalgia, back pain and osteoarthritis were more prevalent among the rheumatic disease cohorts, while diabetes was more prevalent among the HTN cohorts. Liver disease prevalence was low among all cohorts. Rheumatic disease cohorts had higher mean outpatient and primary care visits compared with matched HTN cohorts, while mean emergency department visits and hospitalisations were similar between the cohorts. Physical therapy, NSAIDs and COXIB use were more prevalent among rheumatic disease cohorts. Baseline use of any opioids was higher among the rheumatic disease cohorts while the use of naloxone, and methadone were low among all cohorts. Biologic DMARD use was prevalent among 21\% of patients with RA, $53 \%$ of patients with AS and $43 \%$ of patients with PsA.

During 1-year follow-up, rates of long-term opioid use were higher among all rheumatic disease cohorts compared with their respective HTN cohorts (figure 2). The rate of long-term opioid prescription during follow-up was the highest among AS (25\%), followed by RA (19\%), SLE (16\%) and PsA (15\%), while the rates among the matched HTN cohorts ranged from $5 \%$ to $6 \%$. Among the patients with long-term opioid use during follow-up, the proportion of new opioid users who had no history of opioid use during baseline ranged from $5 \%$ to $8 \%$ in the prevalent disease cohorts. Over the duration of the study, opioid prescriptions had an increase in trend in all cohorts, with a significant increase for RA, PsA and the combined HTN cohorts (figure 3).

The rates of other measures of opioid use including any opioid prescription, receipt of $\geq 3$ types of opioids, immediate-acting and extended-acting opioid prescriptions were similarly higher for the rheumatic disease cohorts and highest for AS (table 2) compared with the HTN cohorts. Overall, among patients with any opioid use, the mean number of days of opioid supply was higher among the rheumatic disease cohorts compared with the HTN cohorts, and the median cumulative morphine equivalent dose per day was lower in the rheumatic disease cohorts compared with each respective HTN cohort (table 2).

For the RA cohort, the rates of those receiving longterm opioids were higher among those receiving biologic DMARDs $(22 \%$ vs $18 \%, \mathrm{p}<0.0001)$. The rates of those receiving long-term opioids was similar among those receiving biologic DMARDs versus no biologic DMARDs in the AS (24\% vs $25 \%, \mathrm{p}=0.32)$ and PsA (15\% vs $15 \%$, 
Table 1 Baseline patient characteristics and comorbid conditions

\begin{tabular}{|c|c|c|c|c|c|}
\hline & RA & SLE & PsA & AS & Combined HTN \\
\hline & $\mathrm{n}=181710$ & $n=45834$ & $\mathrm{n}=30307$ & $\mathrm{n}=7686$ & $n=265537$ \\
\hline \multicolumn{6}{|l|}{ Demographics } \\
\hline Age (SD), years & $55.3(13.1)$ & $47.1(13.1)$ & $49.7(11.5)$ & $44.6(12.0)$ & $53.0(13.4)$ \\
\hline$\%$ female & 77 & 91 & 51 & 39 & 75 \\
\hline \multicolumn{6}{|l|}{ Region of residence (\%) } \\
\hline Northeast & 16 & 16 & 20 & 15 & 15 \\
\hline Midwest & 27 & 21 & 24 & 21 & 25 \\
\hline South & 39 & 42 & 39 & 38 & 45 \\
\hline West & 19 & 20 & 17 & 26 & 15 \\
\hline \multicolumn{6}{|l|}{ Comorbidities (\%) } \\
\hline Smoking & 4 & 4 & 3 & 4 & 4 \\
\hline Alcohol use & $<1$ & $<1$ & 1 & 1 & 1 \\
\hline Substance use & 1 & 1 & 1 & 1 & 1 \\
\hline Depression & 9 & 12 & 10 & 12 & 9 \\
\hline Fibromyalgia & 10 & 16 & 8 & 12 & 3 \\
\hline Back pain & 6 & 7 & 7 & 16 & 5 \\
\hline Migraine headache & 3 & 7 & 3 & 4 & 4 \\
\hline Osteoarthritis & 31 & 17 & 25 & 20 & 11 \\
\hline Hypertension & 36 & 35 & 33 & 26 & 100 \\
\hline Diabetes & 13 & 9 & 14 & 8 & 20 \\
\hline Chronic kidney disease & 2 & 8 & 2 & 2 & 3 \\
\hline Coronary heart disease & 9 & 7 & 7 & 5 & 10 \\
\hline Heart failure & 2 & 3 & 1 & 1 & 3 \\
\hline $\begin{array}{l}\text { Chronic obstructive } \\
\text { pulmonary disease }\end{array}$ & 3 & 12 & 2 & 2 & 3 \\
\hline Asthma & 7 & 8 & 5 & 5 & 6 \\
\hline Sleep apnea & 2 & 2 & 2 & 2 & 2 \\
\hline Liver disease & 3 & 4 & 4 & 3 & 3 \\
\hline Viral hepatitis & 1 & 1 & 1 & 1 & 1 \\
\hline HIV & $<1$ & $<1$ & $<1$ & $<1$ & $<1$ \\
\hline Any fracture & 5 & 4 & 4 & 4 & 3 \\
\hline $\begin{array}{l}\text { Surgery (MSK, } \\
\text { cardiovascular, GI, GU) }\end{array}$ & 7 & 10 & 5 & 5 & 8 \\
\hline Tooth surgery & $<1$ & $<1$ & $<1$ & $<1$ & $<1$ \\
\hline \multicolumn{6}{|l|}{ Healthcare utilisation } \\
\hline Mean outpatient visits (SD) & $10.5(6.9)$ & $11.5(7.7)$ & $9.8(6.4)$ & $10.2(6.9)$ & $6.7(5.2)$ \\
\hline Mean primary care visits (SD) & $5.0(6.4)$ & $5.5(7.5)$ & $4.1(5.0)$ & $4.0(4.9)$ & $4.3(5.0)$ \\
\hline Mean ED visits (SD) & $0.4(1.1)$ & $0.7(1.7)$ & $0.3(1.1)$ & $0.4(1.1)$ & $0.5(1.2)$ \\
\hline Mean hospitalisations (SD) & $0.2(0.5)$ & $0.3(0.7)$ & $0.1(0.4)$ & $0.1(0.5)$ & $0.2(0.5)$ \\
\hline Physical therapy (\%) & 15 & 14 & 15 & 22 & 10 \\
\hline \multicolumn{6}{|l|}{ Medication use (\%) } \\
\hline NSAIDs & 46 & 35 & 46 & 53 & 23 \\
\hline COXIB & 16 & 8 & 12 & 15 & 5 \\
\hline Benzodiazepine & 17 & 21 & 16 & 18 & 16 \\
\hline SSRI & 17 & 19 & 17 & 16 & 16 \\
\hline
\end{tabular}

Continued 
Table 1 Continued

\begin{tabular}{|c|c|c|c|c|c|}
\hline & RA & SLE & PsA & AS & Combined HTN \\
\hline SNRI & 7 & 10 & 7 & 9 & 5 \\
\hline Bupropion & 4 & 6 & 5 & 5 & 4 \\
\hline Gabapentin & 7 & 8 & 5 & 7 & 4 \\
\hline Pregabalin & 3 & 4 & 2 & 4 & 1 \\
\hline csDMARD & 76 & 80 & 55 & 34 & 1 \\
\hline bDMARD & 21 & 1 & 43 & 53 & $<1$ \\
\hline Glucocorticoid & 55 & 55 & 35 & 38 & 14 \\
\hline $\begin{array}{l}\text { Median cumulative } \\
\text { glucocorticoid dose in mg } \\
\text { (IQR) }\end{array}$ & 720 (1300) & 984 (1870) & 400 (816) & 420 (981) & $180(300)$ \\
\hline Naloxone & $<1$ & $<1$ & $<1$ & $<1$ & $<1$ \\
\hline Methadone & $<1$ & 1 & $<1$ & 1 & $<1$ \\
\hline $\begin{array}{l}\text { Any opioid (including } \\
\text { methadone) }\end{array}$ & 48 & 46 & 41 & 52 & 31 \\
\hline
\end{tabular}

AS, ankylosing spondylitis; bDMARD, biologic disease-modifying antirheumatic drug; COXIB, COX-2 inhibitors; csDMARD, conventional synthetic disease-modifying antirheumatic drug; GI, gastrointestinal; GU, genitourinary; MSK, musculoskeletal; NSAID, non-steroidal antiinflammatory drugs; PsA, psoriatic arthritis; RA, rheumatoid arthritis; SLE, systemic lupus erythematosus; SNRI, serotonin and norepinephrine reuptake inhibitors; SSRI, selective serotonin reuptake inhibitors.

$\mathrm{p}=1.0$ ) cohorts. Among the SLE cohort, patients receiving only hydroxychloroquine had significantly lower rates of opioid prescriptions compared with those receiving other immunosuppressants ( $15 \%$ vs $20 \%, \mathrm{p}<0.0001)$.

In our multivariable model with all cohorts included, AS was associated with the highest relative risk of opioid prescription, when adjusted for baseline demographics, comorbidities, medications and healthcare utilisation patterns (table 3). Compared with HTN as reference, the relative risk of receiving long-term opioid prescriptions was 2.73 for AS (95\% CI 2.60 to 2.87 ), followed by relative risk of 2.21 (95\% CI 2.16 to 2.25) for RA. In sensitivity analysis, patients with AS with baseline biologic DMARD use had adjusted relative risk of 2.94 (95\% CI 2.54 to 3.41) of receiving long-term opioids compared with matched

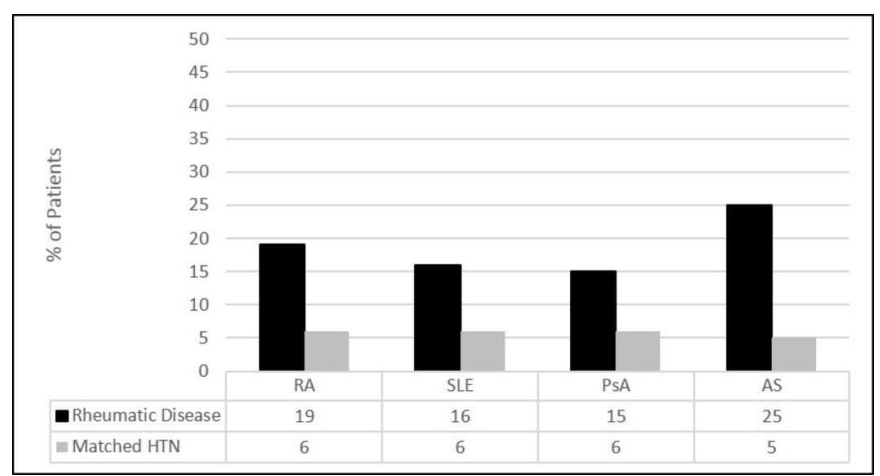

Figure 2 Long-term ( $>90$ days) opioid prescription for each rheumatic disease and matched HTN cohort $p$ value for each comparison between rheumatic disease and matched HTN <0.0001. AS, ankylosing spondylitis; HTN, hypertension; PsA, psoriatic arthritis; RA, rheumatoid arthritis; SLE, systemic lupus erythematosus.
HTN controls, while the relative risk of receiving longterm opioids compared with matched HTN controls in patients with AS without baseline biologic DMARD use was 3.48 (95\% CI 2.85 to 4.26 ).

\section{DISCUSSION}

Prescription opioid use has increased significantly in the USA in the past two decades, coinciding with a rise in opioid related deaths and prompting concerns regarding prescribing and use patterns. ${ }^{18}$ Patients with rheumatic diseases often present with pain as the predominant complaint, and comprise disease populations that may be at higher risk of opioid use. ${ }^{1527}$ In this US nationwide population-based study from 2003 to 2014, we found a

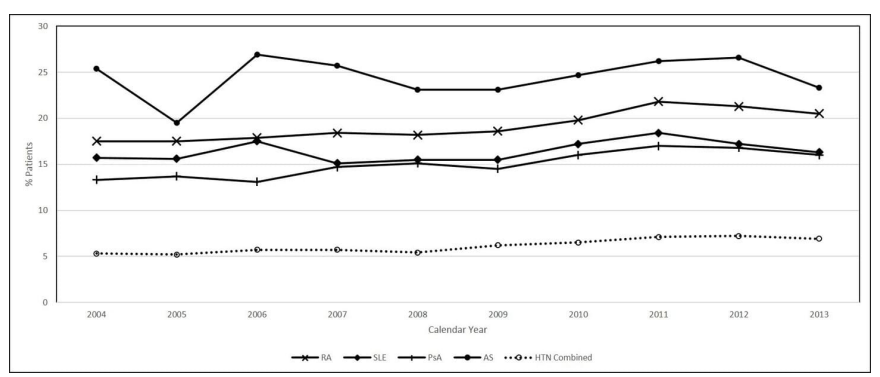

Figure 3 Percentage of patients receiving long-term $(\geq 90$ days) opioid prescriptions per index date calendar year. $P$ value for trend $<0.05$ for RA, PsA and HTN combined; linear regression parameter estimates $(95 \% \mathrm{Cl})$ : RA 0.471 ( 0.283 to $0.659)$, SLE 0.166 (-0.098 to 0.430$)$, PsA 0.417 (0.251 to 0.583 ), AS 0.147 ( -0.447 to 0.742 ) and HTN 0.234 (0.156 to 0.312). AS, ankylosing spondylitis; HTN, hypertension; PsA, psoriatic arthritis; RA, rheumatoid arthritis; SLE, systemic lupus erythematosus. 
Table 2 Opioid prescription rates for each rheumatic disease and matched HTN cohorts

\begin{tabular}{lllllllll}
\hline & \multicolumn{3}{c}{ Matched } & \multicolumn{2}{c}{ Matched } & \multicolumn{2}{c}{ Matched } & \multicolumn{2}{c}{ Matched } \\
& RA & HTN & SLE & HTN & PsA & HTN & AS & HTN \\
\hline \% on any opioids & 46 & 32 & 46 & 33 & 41 & 31 & 50 & 30 \\
\% on >3 types of opioids & 4 & 2 & 5 & 2 & 3 & 2 & 6 & 2 \\
\% on immediate-acting opioid & 46 & 32 & 46 & 33 & 41 & 31 & 49 & 30 \\
\% on extended-acting opioid & 5 & 2 & 6 & 2 & 4 & 2 & 9 & 1 \\
Median cumulative MED per day among & 30.0 & 33.3 & 30.6 & 33.3 & 31.5 & 34.1 & 33.0 & 35.0 \\
patients on opioids (mg/day, IQR) & $(26.3)$ & $(26.8)$ & $(27.5)$ & $(26.7)$ & $(29.0)$ & $(25.6)$ & $(32.3)$ & $(25.0)$ \\
\hline
\end{tabular}

AS, ankylosing spondylitis; HTN, hypertension; MED, morphine-equivalent dose; PsA, psoriatic arthritis; RA, rheumatoid arthritis; SLE systemic lupus erythematosus.

higher prevalence of long-term prescription opioid use among patients with rheumatic diseases (RA, SLE, PsA and AS) compared with age-matched and sex-matched patients with HTN without these rheumatic diseases. Furthermore, we found that the proportion of patients receiving prescription opioids was highest among AS, and AS cohort status was associated with the highest risk of receiving long-term opioids when adjusted for baseline covariates, comorbidities, medications and healthcare utilisation patterns.

Although the finding of higher opioid use in patients with rheumatic disease than HTN is not surprising, our study assessed the prevalence and relative risk of prescription opioid use in RA, SLE, PsA and AS populations. While this is the first study comparing opioid prescriptions among patients with SLE, PsA and AS to patients without these diseases, the findings of this study are consistent with previous studies that have reported higher rates of opioid prescriptions among patients with RA compared

Table 3 Relative risk of receiving long-term opioid prescriptions compared with HTN controls without rheumatic disease

\section{Relative risk ${ }^{\star}$ of receiving long-term opioid prescriptions}

\begin{tabular}{ll}
\hline HTN & 1.00 (ref) \\
RA & $2.21(2.16-2.25)$ \\
SLE & $1.82(1.77-1.88)$ \\
PSA & $1.94(1.87-2.00)$ \\
AS & $2.73(2.60-2.87)$ \\
\hline
\end{tabular}

*Each multivariable model adjusted for age at index date, sex, calendar year of index date, baseline characteristics of region of residence, alcoholism, smoking, substance use, depression, fibromyalgia, back pain, migraine headache, osteoarthritis, chronic kidney disease, liver disease, tooth extraction, MSK surgery, cardiac/digestive/gyn surgery, combined comorbidity score, physician visits, hospitalisations, ED visits, use of NSAIDs, cyclooxygenase-2 inhibitors, benzodiazepines, SSRI, SNRI, gabapentin, pregabalin, cumulative glucocorticoid dose and cohort status of RA, SLE, PsA, AS with hypertension cohort as reference. AS, ankylosing spondylitis; HTN, hypertension; PsA, psoriatic arthritis; RA, rheumatoid arthritis; RR, relative risk; SLE, systemic lupus erythematosus. with non-RA patients. ${ }^{16}{ }^{17}$ In a population-based study in Minnesota of 501 patients with RA and 532 non-RA patients from 2005 to 2014, the rate of total opioid use was higher in patients with RA ( $40 \%$ for RA vs $24 \%$ for non-RA). ${ }^{17}$ Chronic opioid use, defined as $\geq 60$ days in this study, was also higher among patients with RA (12\% for RA vs $4 \%$ non-RA), and patients with RA were 1.5 times more likely to use any opioids. Additionally, RA was associated with prolonged opioid use in a nationwide study of Medicare recipients. ${ }^{28}$ In addition to RA, our multivariable models showed higher risk of prescription opioid use among SLE, PsA and AS rheumatic disease cohorts, after adjusting for other covariates that have been reported to be associated with opioid use such as substance abuse, back pain and fibromyalgia. ${ }^{18} 28$

According to the US Centers for Disease Control and Prevention, use of prescription opioids peaked in 2010, was stable between 2010-2012 then declined through $2015^{1}$. This trend coincided with increasing scrutiny of prescription of opioid analgesics for non-cancer related pain, with a shift towards tighter regulation to curb excessive opioid prescribing including the institution of prescription monitoring programs. ${ }^{29-31}$ The data regarding rates of long-term opioid use in RA has been mixed. A recent study of patients with RA in the Corrona registry showed doubling of chronic opioid use (defined as $\geq 2$ consecutive reports of opioid use) from $7.4 \%$ in 2002 to $16.9 \%$ in 2015. In contrast, a study of US Medicare patients with RA showed that rates decreased from 2010 to 2014, similar to the trend reported in the general population. ${ }^{18}{ }^{19}$ Similar temporal patterns were seen among the HTN, RA and PsA cohorts through these years in our study which likely reflects the general prescribing patterns of opioids rather than being specific to rheumatic disease populations.

Interestingly, although prescription opioid use (measured by dispensing of any opioid prescriptions, long-term prescriptions, immediate-acting or extended-acting opioids, and $\geq 3$ types of opioids) was higher for the rheumatic disease cohorts compared with the HTN cohorts without rheumatic disease, the median cumulative morphine equivalent doses among those receiving opioid prescriptions were lower among the rheumatic disease cohorts. This suggests that while patients with rheumatic 
diseases receive a greater number of opioid prescriptions the average doses appear to be lower. Although the reason for this finding is unclear, indications and patterns of prescription opioid use may be different among patients with rheumatic diseases compared with the HTN cohort and further research is needed to determine the association between the use of opioids and the risk of adverse clinical outcomes in patients with underlying rheumatic diseases.

This study demonstrates that long-term prescription opioid use, as well as any opioid use, immediate-acting, extended-acting and $\geq 3$ types of opioid prescriptions were all highest among patients with AS. Long-term prescription opioid use was prevalent in $25 \%$ of patients with AS which is consistent with findings published in a recent study of 11945 patients with AS identified by ICD-9 codes in Truven Marketscan commercial claims where $23.5 \%$ patients had chronic opioid use. ${ }^{32}$ One possible explanation for the high prevalence of long-term opioid use in AS is that chronic low back pain, which is associated with opioid prescription ${ }^{33-35}$, is more common among patients with AS and more debilitating than pain in other locations. However, even after adjusting for comorbidities and potential confounders of opioid use, including back pain, AS cohort status was associated with the highest relative risk of opioid prescription, although back pain in patients with AS may not necessarily be coded separately.

Another potential explanation for this finding is that patients with AS may continue to experience pain regardless of treatment or may have experienced delays in treatment which could affect response to DMARDs, as opioid use seems to be associated with lower odds of persistence on tumor necrosis factor (TNF) inhibitors after initiation in $\mathrm{AS}^{36}$ Interestingly, in a multi-centre cohort of 706 patients with AS, opioid use was found to be associated with subjective measures of disease by the patient, but not with objective measures of disease by erythrocyte sedimentation rate, C-reactive protein or baseline radiographic severity. ${ }^{37}$ Additionally, opioid use was associated with older age, smoking and psychiatric medications, while slightly negatively associated with TNF inhibitor use (multivariable OR $0.7,95 \%$ CI 0.5 to 1.0 ).

Although it is not possible to assess disease severity or activity in this claims-based study, it is notable that whereas patients with RA on biologic therapy had slightly higher rates of opioid use possibly due to worse disease, AS cohort had no difference in long-term opioid prescriptions based on biologic DMARD use at baseline. Furthermore, patients with AS who did not receive biologic DMARDs during baseline had 3.48 (95\% CI 2.85 to 4.26 ) relative risk of long-term opioid use compared with matched HTN controls, and the relative risk in patients with AS who received biologic DMARDs during the baseline period was 2.94 (95\% CI 2.54 to 3.41) compared with matched HTN controls. This raises the possibility that although over half of the patients with AS were on biologic DMARD therapy, some patients with AS may be receiving symptomatic treatment with opioids rather than being treated with disease targeted therapies, or may experience delay in treatment or access to care. Patients with AS may also experience delays in initiating disease-modifying treatment until radiographic progression of disease, as TNF inhibitors have not been approved for non-radiographic axial spondyloarthropathy in the USA during the study period. ${ }^{38}$ Further investigation addressing treat-to-target strategies on pain and opioid use may identify strategies for reducing long-term opioid use and prescribing practices in patients with AS.

Our study has several strengths as a large nationwide study of opioid prescribing among four different rheumatic disease populations with age-matched and sex-matched comparator groups. We obtained detailed analysis of opioid prescriptions dispensed and demonstrated that while opioid prescription rates by any opioids, long-term, $>3$ types, immediate-acting and extended-acting opioids were all higher for the rheumatic disease cohorts, and highest among AS, the morphine equivalent dose per day was lower among the rheumatic disease cohorts compared with the matched non-rheumatic disease cohorts.

There may be potential misclassification of disease cohorts, although we minimised this possibility by requiring at least two ICD-9 codes with a disease specific medication to identify our cohorts. This algorithm has been validated for RA, with a positive predictive value of $>86 \%{ }^{39}$ and has been used in patients with SLE. ${ }^{40}$ The algorithm has a $>80 \%$ positive predictive value for $\mathrm{PsA}^{41}$ but has not been validated for identifying patients with AS, and is likely more specific than the validated algorithm for identifying patients with HTN using only ICD-9 codes without the use of a prescription. ${ }^{42}$ Our inclusion criteria required at least 12 months of baseline and follow-up period which limits the generalisability of our results to a cohort of survivors.

We used a matched cohort design to compare each rheumatic disease cohort with patients without rheumatic diseases of similar age and sex, and selected patients with HTN, a chronic condition not characterised by pain, with routine outpatient visits. While patients with HTN may not be representative of all patients without rheumatic disease, and still had fewer outpatient visits than the rheumatic disease groups at baseline, the proportion of patients who received prescription opioids in the HTN group in our study are in line with reported population estimates. ${ }^{28}{ }^{43}$ Furthermore, while our disease cohorts were prevalent cohorts, future studies in incident disease cohorts may be able to look into patterns of opioid use before and after disease diagnosis and the potential effect of disease-specific treatments.

Additionally, our limitations include lack of information regarding pain level or disease severity and duration, as severe pain, high disease activity and disability in patients with RA have been found to be associated with chronic opioid use. ${ }^{19}$ In an attempt to adjust for disease severity, we included cumulative glucocorticoid use during baseline period in our multivariable model as a surrogate to adjust 
for disease severity, although results remained similar to models that did not include glucocorticoid use. Our outcome was measured by opioid prescriptions dispensed using billing claims, which may not accurately reflect actual intake of opioids. Diversion of opioids is thought to be common, although the true rates and whether there is a difference in behaviour pattern of opioid misuse and diversion among patients with rheumatic diseases compared with the general population is unknown. ${ }^{4-47}$

While we included covariates such as obesity, smoking and substance use, the measured rates were low, as these comorbidities are likely under-reported in claims data. Other covariates that are likely associated with opioid use which were not available in our database include race/ethnicity data, cannabinoid use and physician characteristics. ${ }^{18} 4849$ Additionally, a multidisciplinary approach to pain, which includes patient education, exercise programmes and psychological or social interventions, is an effective part of pain management in inflammatory arthritis that we were unable to assess in our claims-based study. ${ }^{27}$

In this large US nationwide study of opioid prescriptions among a total of 531074 patients, patients with RA, SLE, PsA and AS had higher rates of opioid prescriptions compared with age-matched and sex-matched patients with HTN. Furthermore, AS was associated with the highest relative risk of receiving long-term prescription opioids. Although pain which is a common complaint among patients with rheumatic diseases may be the reason for this observed difference, further studies looking at disease activity and effectiveness of treat-to-target strategies in treatment of rheumatic diseases in decreasing long-term opioid use may better elucidate the cause of this finding and identify strategies to reduce prescribing and risk of opioids.

Contributors All authors were involved in drafting the article or revising it critically for important intellectual content, and all authors approved the final version to be submitted for publications. Drs. SC and SCK had full access to all of the data in the study and take responsibility for the integrity for the data and the accuracy of the data analysis. Study conception and design: SC, CF, GB, YL, RD, SCK. Acquisition of data:SC, GB, SCK. Analysis and interpretation of data: SC, CF, GB, YL, RD, SCK.

Funding This study was supported by the Division of Pharmacoepidemiology and Pharmacoeconomics, Brigham and Women's Hospital, Harvard Medical School.

Competing interests None declared.

Patient consent for publication Not required.

Ethics approval The Institutional Review Board of the Brigham and Women's Hospital approved this study.

Provenance and peer review Not commissioned; externally peer reviewed. Data sharing statement No additional data are available.

Open access This is an open access article distributed in accordance with the Creative Commons Attribution Non Commercial (CC BY-NC 4.0) license, which permits others to distribute, remix, adapt, build upon this work non-commercially, and license their derivative works on different terms, provided the original work is properly cited, appropriate credit is given, any changes made indicated, and the use is non-commercial. See: http://creativecommons.org/licenses/by-nc/4.0/.

\section{REFERENCES}

1. Guy GP, Zhang K, Bohm MK, et al. Vital Signs: Changes in Opioid Prescribing in the United States, 2006-2015. MMWR Morb Mortal Wkly Rep 2017;66:697-704.
2. Nelson LS, Perrone J. Curbing the opioid epidemic in the United States: the risk evaluation and mitigation strategy (REMS). JAMA 2012;308:457-8.

3. Busse JW, Wang L, Kamaleldin M, et al. Opioids for Chronic Noncancer Pain. JAMA 2018;320:2448.

4. Chou R, Turner JA, Devine EB, et al. The effectiveness and risks of long-term opioid therapy for chronic pain: a systematic review for a National Institutes of Health Pathways to Prevention Workshop. Ann Intern Med 2015;162:276.

5. Moore RA, McQuay HJ. Prevalence of opioid adverse events in chronic non-malignant pain: systematic review of randomised trials of oral opioids. Arthritis Res Ther 2005;7:R1046-51.

6. Edlund MJ, Martin BC, Russo JE, et al. The role of opioid prescription in incident opioid abuse and dependence among individuals with chronic noncancer pain: the role of opioid prescription. Clin J Pain 2014;30:557-64.

7. Rudd RA, Seth P, David F, et al. Increases in Drug and OpioidInvolved Overdose Deaths - United States, 2010-2015. MMWR Morb Mortal Wkly Rep 2016;65:1445-52.

8. Bohnert AS, Valenstein M, Bair MJ, et al. Association between opioid prescribing patterns and opioid overdose-related deaths. JAMA 2011;305:1315-21.

9. Wiese AD, Griffin MR, Stein CM, et al. Opioid Analgesics and the Risk of Serious Infections Among Patients With Rheumatoid Arthritis: A Self-Controlled Case Series Study. Arthritis Rheumatol 2016;68:323-31.

10. Saunders KW, Dunn KM, Merrill JO, et al. Relationship of opioid use and dosage levels to fractures in older chronic pain patients. $J$ Gen Intern Med 2010;25:310-5.

11. Acurcio FA, Moura CS, Bernatsky S, et al. Opioid Use and Risk of Nonvertebral Fractures in Adults With Rheumatoid Arthritis: A Nested Case-Control Study Using Administrative Databases. Arthritis Rheumatol 2016;68:83-91.

12. Solomon DH, Rassen JA, Glynn RJ, et al. The comparative safety of analgesics in older adults with arthritis. Arch Intern Med 2010;170:170.

13. Heiberg T, Finset A, Uhlig T, et al. Seven year changes in health status and priorities for improvement of health in patients with rheumatoid arthritis. Ann Rheum Dis 2005;64:191-5.

14. Lee YC. Effect and treatment of chronic pain in inflammatory arthritis. Curr Rheumatol Rep 2013;15:300.

15. Kwoh CK, Ibrahim SA. Rheumatology patient and physician concordance with respect to important health and symptom status outcomes. Arthritis Rheum 2001;45:372-7.

16. Solomon DH, Avorn J, Wang PS, et al. Prescription opioid use among older adults with arthritis or low back pain. Arthritis Rheum 2006;55:35-41.

17. Zamora-Legoff JA, Achenbach SJ, Crowson CS, et al. Opioid use in patients with rheumatoid arthritis 2005-2014: a population-based comparative study. Clin Rheumatol 2016;35:1137-44.

18. Curtis JR, Xie F, Smith C, et al. Changing Trends in Opioid Use Among Patients With Rheumatoid Arthritis in the United States. Arthritis Rheumatol 2017;69:1733-40.

19. Lee YC, Kremer J, Guan H, et al. Chronic Opioid Use in Rheumatoid Arthritis: Prevalence and Predictors. Arthritis Rheumatol 2019;71:670-7.

20. Parsells Kelly J, Cook SF, Kaufman DW, et al. Prevalence and characteristics of opioid use in the US adult population. Pain 2008;138:507-13.

21. Campbell Cl, Weisner C, Leresche L, et al. Age and gender trends in long-term opioid analgesic use for noncancer pain. Am J Public Health 2010;100:2541-7.

22. Desai RJ, Rao JK, Hansen RA, et al. Tumor necrosis factor- $\alpha$ inhibitor treatment and the risk of incident cardiovascular events in patients with early rheumatoid arthritis: a nested case-control study. $J$ Rheumatol 2014;41:2129-36.

23. Kim SC, Schneeweiss S, Myers JA, et al. No differences in cancer screening rates in patients with rheumatoid arthritis compared to the general population. Arthritis Rheum 2012;64:3076-82.

24. Gagne JJ, Glynn RJ, Avorn J, et al. A combined comorbidity score predicted mortality in elderly patients better than existing scores. $J$ Clin Epidemiol 2011;64:749-59.

25. Centers for Disease Control and Prevention. Opioid Morphine Equivalent Conversion Factors. 2015 https://www.cms.gov/ Medicare/Prescription-Drug-Coverage/PrescriptionDrugCovContra/ Downloads/Opioid-Morphine-EQ-Conversion-Factors-March-2015. pdf (Accessed 1 Jan 2017).

26. Schreiber-Gregory DN, Jackson HM. Multicollinearity: What Is It, Why Should We Care, and How Can It Be Controlled? https://support.sas. com/resources/papers/proceedings17/1404-2017.pdf (Accessed 8 Ap 2019) 
27. Geenen R, Overman CL, Christensen R, et al. EULAR recommendations for the health professional's approach to pain management in inflammatory arthritis and osteoarthritis. Ann Rheum Dis 2018;77:797-807.

28. Kuo Y-F, Raji MA, Chen N-W, et al. Trends in Opioid Prescriptions Among Part D Medicare Recipients From 2007 to 2012. Am J Med 2016;129:221.e21-30.

29. Häuser W, Fitzcharles MA. Pain management: Opioids for RA: a clinical dilemma. Nat Rev Rheumatol 2017;13:521-2.

30. Dowell D, Haegerich TM, Chou R. CDC Guideline for Prescribing Opioids for Chronic Pain - United States, 2016. MMWR Recommendations and Reports 2016;65:1-49.

31. Davis CS, Pierce M, Dasgupta N. Evolution and convergence of state laws governing controlled substance prescription monitoring programs, 1998-2011. Am J Public Health 2014;104:1389-95.

32. Sloan VS, Sheahan A, Stark JL, et al. Opioid Use in Patients with Ankylosing Spondylitis Is Common in the United States: Outcomes of a Retrospective Cohort Study. J Rheumatol 2019:jrheum.180972.

33. Morasco BJ, Duckart JP, Carr TP, et al. Clinical characteristics of veterans prescribed high doses of opioid medications for chronic non-cancer pain. Pain 2010;151:625-32.

34. Deyo RA, Smith DH, Johnson ES, et al. Opioids for back pain patients: primary care prescribing patterns and use of services. J Am Board Fam Med 2011;24:717-27.

35. Gore M, Sadosky A, Stacey BR, et al. The Burden of Chronic Low Back Pain. Spine 2012;37:E668-E677.

36. Hunter T, Schroeder K, Sandoval D, et al. Persistence, Discontinuation, and Switching Patterns of Newly Initiated TNF Inhibitor Therapy in Ankylosing Spondylitis Patients in the United States. Rheumatol Ther 2019;6:207-15.

37. Dau JD, Lee M, Ward MM, et al. Opioid Analgesic Use in Patients with Ankylosing Spondylitis: An Analysis of the Prospective Study of Outcomes in an Ankylosing Spondylitis Cohort. J Rheumatol 2018;45:188-94.

38. Deodhar A, Reveille JD, van den Bosch F, et al. The concept of axial spondyloarthritis: joint statement of the spondyloarthritis research and treatment network and the Assessment of SpondyloArthritis international Society in response to the US Food and Drug Administration's comments and concerns. Arthritis Rheumatol 2014;66:2649-56.

39. Kim SY, Servi A, Polinski JM, et al. Validation of rheumatoid arthritis diagnoses in health care utilization data. Arthritis Res Ther 2011;13:R32

40. Feldman $\mathrm{CH}$, Marty FM, Winkelmayer WC, et al. Comparative Rates of Serious Infections Among Patients With Systemic Lupus Erythematosus Receiving Immunosuppressive Medications. Arthritis Rheumatol 2017;69:387-97.

41. Ford J, MacFarlane L, Tong A, et al. Identification of Psoriatic Arthritis Using an Administrative Claims-Based Algorithm. ACR/AHRP Annu Meet 2018.

42. Tu K, Campbell NR, Chen ZL, et al. Accuracy of administrative databases in identifying patients with hypertension. Open Med 2007;1:e18-26.

43. Hoots BE, Xu L, Kariisa M, et al. Annual Surveillance Report of DrugRelated Risks and Outcomes United States. 2018 https://www.cdc. gov/drugoverdose/pdf/pubs/2018-cdc-drug-surveillance-report.pdf (Accessed 29 Mar 2019).

44. Seamans MJ, Carey TS, Westreich DJ, et al. Association of Household Opioid Availability and Prescription Opioid Initiation Among Household Members. JAMA Intern Med 2018;178:102.

45. Clark DJ, Schumacher MA. America's Opioid Epidemic. Anesthesia \& Analgesia 2017;125:1667-74.

46. McDonald DC, Carlson KE. Estimating the prevalence of opioid diversion by "doctor shoppers" in the United States. PLoS One 2013;8:e69241.

47. Dart RC, Surratt HL, Cicero TJ, et al. Trends in opioid analgesic abuse and mortality in the United States. N Engl J Med 2015;372:241-8.

48. Bachhuber MA, Saloner B, Cunningham CO, et al. Medical cannabis laws and opioid analgesic overdose mortality in the United States, 1999-2010. JAMA Intern Med 2014:174:1668.

49. Choo EK, Feldstein Ewing SW, Lovejoy TI, et al. Opioids Out, Cannabis In: Negotiating the Unknowns in Patient Care for Chronic Pain. JAMA 2016;316:1763-4. 